\title{
Small-bowel obstruction secondary to obturator hernia
}

\author{
Fu-Zong Wu MD, Jer-Shyung Huang MD, Chia-Ling Chen MEd
}

Previously published at www.cmaj.ca
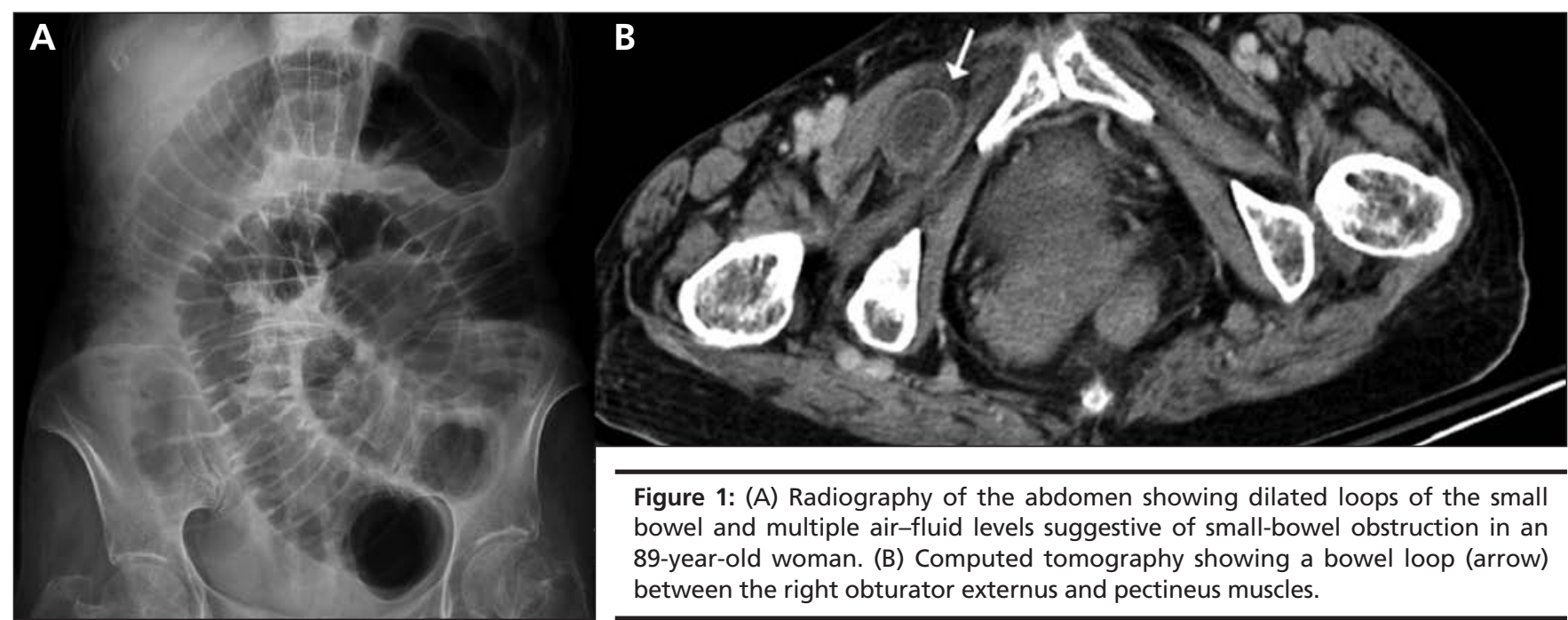

Figure 1: (A) Radiography of the abdomen showing dilated loops of the small bowel and multiple air-fluid levels suggestive of small-bowel obstruction in an 89-year-old woman. (B) Computed tomography showing a bowel loop (arrow) between the right obturator externus and pectineus muscles.

A n 89-year-old woman presented with a three-day history of diffuse abdominal pain and vomiting. She also reported pain in the medial region of her right thigh, which was exacerbated by adduction. She had no history of abdominal surgery. On physical examination, she had a distended abdomen with decreased bowel sounds. No palpable inguinal mass or hernia was found. A radiograph of the abdomen showed dilated loops of small bowel and multiple airfluid levels suggestive of small-bowel obstruction (Figure 1A). Computed tomography showed a bowel loop between the right obturator externus and pectineus muscles (Figure 1B), consistent with a diagnosis of strangulated small-bowel obstruction secondary to an obturator hernia. In an emergency laparotomy, the affected bowel was resected and the hernia repaired.The patient's postoperative recovery was uneventful.

An obturator hernia is a rare intra-abdominal hernia, accounting for $1 \%$ of all hernias and $1.6 \%$ of small-bowel obstructions. ${ }^{1}$ Obturator hernias are three times more common on the right side than on the left. Risk factors include age over 70 years, female sex (the female:male ratio is 6:1), multiparity, underweight, chronic constipation, chronic obstructive pulmonary disease and ascites. ${ }^{2}$ The diagnosis of obturator hernia is difficult because symptoms are nonspecific. In most patients, the condi-

From the Department of Radiology (Wu, Huang), Kaohsiung Veterans General Hospital, Kaohsiung, Taiwan; and the Department of Health Education (Chen), National Taiwan Normal University, Taipei, Taiwan

CMAJ 2010. DOI:10.1503/cmaj.092206 tion is diagnosed during exploratory laparotomy for smallbowel obstruction. Pain along the ipsilateral thigh, exacerbated by adduction of the thigh (Howship-Romberg sign), is highly suggestive of an incarcerated obturator hernia and is caused by compression of the obturator nerve by the hernia. ${ }^{2}$

Preoperative diagnosis by computed tomography of the abdomen is important for detecting small-bowel gangrene and for planning surgical intervention. ${ }^{3,4}$ Bedside ultrasonography of the abdomen in the emergency department can also be diagnostic. ${ }^{5}$ Exploratory laparotomy is recommended when bowel strangulation or gangrene is suspected. Minimally invasive laparoscopic repair can be used for nonstrangulated, reducible obturator hernias. Early diagnosis, appropriate imaging and prompt surgical intervention can reduce morbidity and mortality and improve prognosis., ${ }^{3,4}$

This article has been peer reviewed.

Competing interests: None declared.

\section{REFERENCES}

1. Lo CY, Lorentz TG, Lau PW. Obturator hernia presenting as small bowel obstruction. Am J Surg 1994;167:396-8.

2. Chang SS, Shan YS, Lin YJ, et al. A review of obturator hernia and a proposed algorithm for its diagnosis and treatment. World J Surg 2005;29:450-4.

3. Rodríquez-Hermosa JI, Codina-Cazador A, Marto-Genover A, et al. Obturator hernia: clinical analysis of 16 cases and algorithm for its diagnosis and treatment. Hernia 2008;12:289-97.

4. Silva AC, Pimenta M, Guimarães LS. Small bowel obstruction: what to look for. Radiographics 2009;29:423-39.

5. Gilliam A, O’Boyle CJ, Wai D, et al. Ultrasonic diagnosis of strangulated obturator hernia. Eur J Surg 2000;166:420-1. 